\title{
IRIS Y EL FEMINISMO ARISTOCRÁTICO
}

\author{
Bernardo Subercaseaux \\ Universidad de Chile
}

En la década del Centenario (1910), el pensamiento de emancipación de la mujer transita por varias vías. Por una parte el feminismo aristocrático y la corriente estética a través de la cual éste se expresó: al que hemos llamado espiritualismo de vanguardia ${ }^{1}$, movimiento que tuvo en Inés Echeverría a su figura más destacada. Por otra parte, el feminismo laico y mesocrático representado en gran medida por Amanda Labarca, y, en el mundo popular, el feminismo en sectores ácratas y obreros que se manifestó sobre todo en las cobradoras y conductoras de tranvía, mujeres que operaron en el espacio público de la capital entre fines del siglo XIX y 1930.

Con respecto al feminismo aristocrático, sus figuras más destacadas fueron, entre otras, María Luisa Fernández -madre de Vicente Huidobro- Inés Echeverría de Larraín (Iris), Mariana Cox Stuven (Shade), Sara Hubner, Rebeca Matte (la escultora) y Teresa Wilms. Casi todas ellas tuvieron los recursos económicos y el tiempo para dedicarse a las que llamaban "actividades del espíritu". También participaron, colaboraron o dirigieron revistas y agrupaciones destinadas al fomento de la independencia y autonomía de la mujer, estimulando su interés por la educación, el arte y la cultura. Fundaron o participaron en instituciones como el Club de Lectura (1915) o el famoso y controvertido Club de Señoras (1916-1923), institución que incentivó el rol de la mujer y realizó una extensa labor en el campo cultural de elite, situándose en las antípodas de organizaciones conservadoras como la Liga de las Damas Chilenas, institución ésta que percibía en la cultura moderna (sobre todo en el teatro y en el cine, pero también en el hecho de que la mujer ampliara sus horizontes más allá de la casa y la familia), una amenaza para la moral y las buenas costumbres. Revistas como La Familia (publicada por Zig-Zag entre 1910 y 1928), La revista azul (1914-1916), Silueta (1917-1918) y La tribuna ilustrada (1917), de las cuales fueron colaboradoras, tuvieron una estrategia proselitista pero no confrontacional, intentaban vincular los intereses tradicionales de la mujer de elite (la moda, la decoración)

Véase, Bernardo Subercaseaux, Historia de las ideas y la cultura en Chile, Volumen II. Editorial Universitaria, Santiago, 2011. Recurrimos en esta introducción básicamente a ese volumen. 
con los nuevos intereses de la mujer en el mundo moderno (el arte, la lectura, la vida del espíritu). De allí que con frecuencia se perciba en estas publicaciones cierta tensión entre sujeto femenino y sujeto aristocrático, o entre un sujeto femenino tradicional y otro que busca ampliar el horizonte de la mujer hacia la cultura y lo público. Es en esta zona de tensiones del espiritualismo de vanguardia que indaga la brillante entrevista que le hace Amanda Labarca a Iris en la revista La Familia (1915).

Hay que señalar que tanto Iris como el resto de las mencionadas vivían estilos de vida que desafiaban los moldes tradicionales, eran consideradas excéntricas y tildadas -en algunos casos- de inmorales por la sociedad "bien pensante" y el "vecindario decente" de la época. Desde un punto de vista político, a pesar de su filiación aristocrática, casi todas ellas apoyaron a Arturo Alessandri y también al movimiento estudiantil. Mantuvieron sin embargo cierta distancia frente a la política, como señala un editorial de La Revista Azul : "podríamos hablar sobre política pero preferimos no tocarla, pues pensamos como Madame Augol que "la politique est tres peu poetique".

Iris puede considerarse como la figura más representativa del espiritualismo de vanguardia, de esa sensibilidad que percibía la vida del alma como la experiencia más sublime y trascendente, como la única que enaltecía y justificaba la existencia humana. Concebía la morada interior y la profundidad de la vida espiritual como el foco de toda creación y como materia prima del arte. De allí que los géneros preferidos sean la prosa poética y los géneros memorialísticos, los diarios de vida y de viajes, los perfiles y viñetas. Más que por el camino de la razón o de lo meramente descriptivo, recurrían a los presentimientos, a las revelaciones fugitivas e inexplicables, a las sugestiones o las divagaciones nebulosas. Para esta sensibilidad el reino del espíritu no tenía fronteras ni nacionalidad. Son mujeres que practicaban una suerte de ecumenismo espiritual. "No son-escribió Iris- los barcos ni los trenes los que alejan o acercan a los corazones. Son los grados de nuestro desarrollo interior. Por encima de las ciudades chatas o de los altos rascacielos que habitamos, están las ciudades espirituales a que pertenecemos, y cuya legítima ciudadanía no limitan ni las distancias ni las fronteras" ${ }^{2}$. Iris como la mayor parte de las autoras vinculadas a esta tendencia, tuvo una visión crítica y más bien negativa de Chile, situándose en las antípodas de ese nacionalismo que caracterizó las fiestas del Centenario "Por desgracia hay en mi tierra -advierte- una ranciedad de mente, una opacidad de atmósfera, que cuando encontramos algo espontáneo, ligero, preguntamos atónitos si por ventura no hemos (acaso) traspasado la frontera"3.

Es con estas preferencias que interactúa la entrevistadora Amanda Labarca, asumiendo desde una perspectiva mesocrática - con cariz republicano y cívico- un punto de vista crítico que no deja de ser amable. Está consciente de que si bien estas mujeres desafiaban la concepción tradicional y conservadora de lo femenino, en lugar de luchar políticamente ante ella se inclinaban más bien por formas alternativas de intensificar la vida espiritual

2 Iris "La mujer en sus diversos estados" Transcripción de conferencia dictada ante las voluntarias de la Cruz Roja, La Nación, Santiago, 7-1-1923.

Iris, op. cit. 
como la teosofía y el espiritismo. Pero aun así, las percibe como una fuerza positiva en pro de los derechos de la mujer, e intenta atraerlas hacia un feminismo mesocrático y republicano, portador de un proyecto educativo que abarcaba no solo a las mujeres de elite sino también a las capas medias y a todas las mujeres del país. Se trata de ideas y sensibilidades diferentes - pero con algunas pulsiones en común-, es en este contexto de un diálogo entre dos mundos que hay que leer la presente entrevista.

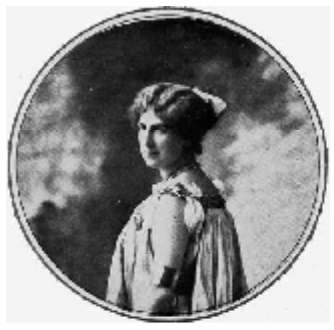

\section{Qla vida del eppiritu}

A pato, 1915

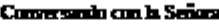

hós Hderain in I min

Buscando el frescon de un autis en la aidez de las meacypsiones dimigs, fuimos, no hors mochas tredes, a

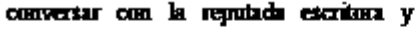
distingridg dima Inés Echereríg de Lamin, cuyos peodísimos Iis e Inés Bello soo, de ciertb, holpitmoles a las lectrons de "FAMIIIA".

Noestra anvessación nn torí nada del Ieportaje Fhígn atedas sns palobngs y las mias ln incitbem

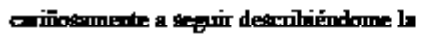
vidr de san Exṕinim

In melmoxulíg de la trode

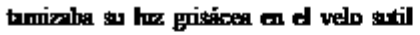
de la neblim Afixer d viruto bineals sass filos an his gantrs, en las ves aterides, an las remas deshojadss de hos äthales, mientras que en ha tilin atmosfier

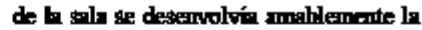
charla secturform

Crmo engarses finns parecian en la aprebada malla de so conversorión los

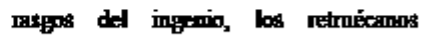
thisperuter La parin pirceser de su deri fivente. Pero no are tolp sus plobras has que me interesthem quiri pendrir a traves de ellos, de sus gestris $y$

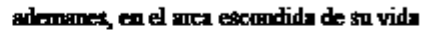
esjimitugl

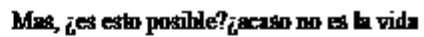

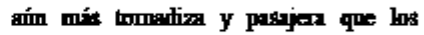
celajes de nii cielo de ofotio?

Puede qu fuer la melmaolía de la estacíinn Las claridndes morilentes de la trade, el frío, y quíén she que atro: fintros deteminhntes de ese mominto, lns que me mostreon a fris comm uns descrintenth de todo: de sal pris, de sey 
modin, de su fiore e igolmante de hs firmes en que hath yqui ha vacigdo los proturtos de sin atre

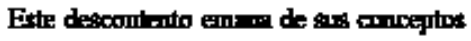
y se revela por ifanl an sasios y punz de inomin Este respo que comstituye

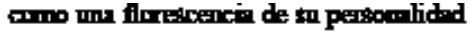
le ha suscifodo mis de una vez destratnes y molestios inmerexidgs hit Es u... mnjer que siente Fofindmente, que vilur con les emocinnes de los demiss qu simpitiza froilmonte cin los otros y

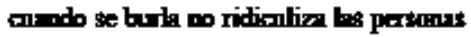
sinn los defiectos. La vaciedad, la Fresumión, d reglln nexio amo la

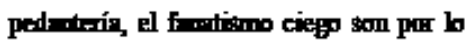

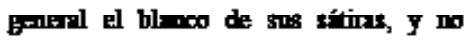
poede neprose que si en vez de describii

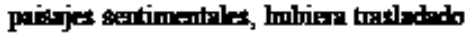
al papel todo ed hmmbismo, el inforio y

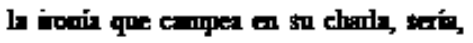
Imin de les mít repundes novelista de Chile, la mís origim y pexirda de todns les atitrs maricanns

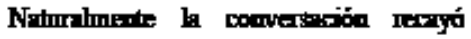
sobes su obry

- ¿Pro qué ha excrito usted en frectis sn último hinar: "Entre Dars Mondes"?
- Porque es el inioma de mi arte: porque

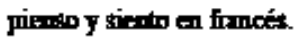

- ¡Qué cosen mís ram! Siendo usted chilon y todnvía ming del más grande de los pramáticos castr|lmos...

- No, no es mo. Voy a coctirle a usted los primcipios de mi vocraión y se convincerí De monthoch y viviendo todrvig en el mitto enchestrminto de la frailin, sentix ye el impulso de excribii, pert me daha curnta tmbirin de lo imantito de semajate impolsir: um mincherch escribiendo, y eseribiendo Ping tural $Y$ no obstante $y$ a pestr de todo, yo sentín inmprios mante la necesidad de dir forma a mis

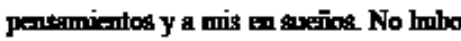
mís que un metio de crnolite mis vehementes deseos con ed ntwall prodor de substraer mis excritos a los comant ins y a las buils, y escili en fimbés. Ins whes extranjugras lleztlon a haxírseme hrbitnales y durate mi vid he redactodo el dimio de mis digs en be lingen que for amgha mík. .

- Entmices, ja netted no le porth el castellmo?

- ¡No, mil rexes m. El cattell or Es Par mí ha lenger de la cocinerp, del 
Friveede, de bes mentes de la crose..Si

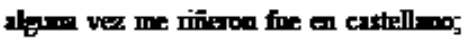
los que me pelas, gracirs a Dink, tambión lo baxen en castrimb... iy netred quibe que bo ande?

- Es que el atista oo debe trovier solo a la iden, ormbien a ln palghe Todo exring purifies, afim y ductiliza el idiomn, y sirndo asi, creo yo que usted tiene bo ohligarión de collive el suyo y ne utro.

$-i^{P}$ in quit?

- Porque el idiome es unn porte del glma

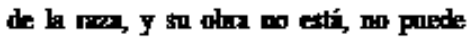
estra aisladx es un producto de un estodo de conciencin de la oloxtivided en m momento de st evolusión, y yrodízxame

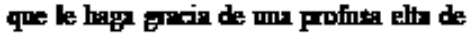
Trie Qre aqui viene al purto--

- Despraciadamente, for timpoco tifon

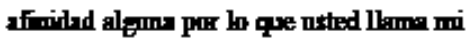
nar Mro mi estipe y no me remmero. In que estuvieron cerea de mí en los ains díctiles de h inforiv y en los aings mingrotos de ba juvintod, ne hiciertin nada por desimolle en mí ets solidarided

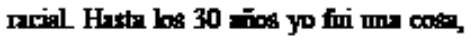
algo que bahía patido limm sim detmedo un ser esclovo y hath

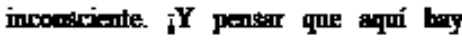
mijeres que no pasco mmore de hos trintp aี̄oㄴ!

- ¿Y la pitria tampoco hubla a sa

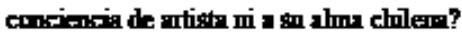

- Mans todquín ¿Qué es la Pain? ¿Quién be puede defin? ¿Por quó ben de ser mís hemanns ming los que ven oculthrse d sol trats de los mores, que los que he virion exrmiderse detris de las montinge A mí bo me edurinon en el amor a be Patrig, ni yo lo he patertido a senti después Amo h Emogr mocho mís que ba Amíniry purque a peser de aquí bay vol monte repiblirss y sucle haber all manrquígs, puede vivirse en ellat ma vidn mít hilhe, mít combirite, menos llew de emedos, de abionos, de pequeīeces; mít amplig, más

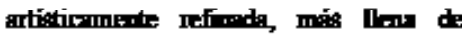
bellen, de ath, de alegrín, de talentor y de persomalidar Todo es mis fingl allí,

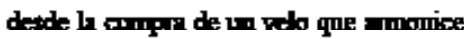
con ed trije, hofa la pohlicarín de un lio y ha rancepción de muxhos otros.

- No rmpinto en modo alyano sus

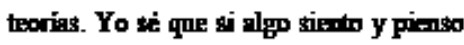

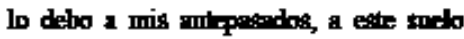
que me nutre, a estes montuins que me cobïgn, a esta hz rada que Es nuestio 
halo y mestio ambicite; me cien tom solid ix de mis cllmpinictas; tm pimitivam:nte adhrida al trrmin que no

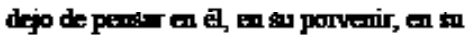
prindera y en sin sopte cadn vez que mis palbluas busan m ero en otris almer Y si todo es difi:terlle aqui, comb usted dice, es porque on angmo bastmite a nuestro pás Pan padmarle de boen grodo los pequrións tancificios que nos impone.

- Pero nil me neqpá usted que el

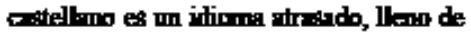
harmibse, tiest, con el cull es impotible akangr les modnlariones del firmexis

- Sí b niefo. Ahí tiene usted a los modereos hablistes, Valle Inctín, por ciminglo. ¿No bo commint ntsed en mestrin del dexil a crolquien de sns

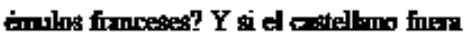
ung lenger en mahexidy, los antistas como usted deberím dirle be flecibitided que usted cree que le hace filth

- ¡Oh, no! ¿For quix se hurí de adoptri unx Irm: mellads curndo puede disponer de mo limin de axero flexible, rexin y firme al mitmo tiempo?

- Porqu la piman es la que usted posee y b tepmind, tione que pedind prestada al rexino.
- Na nos poodtemos de acrado sobe etto...

- Afortmadrmente estmos cieths de concondr en muxhas otres costs iCómn vat solbo?

- jNo me dipg! ¡Si esto es mn hrrra! Con ung impranided de colepiala yo me hahía hecto a h ilsoicon de construime un inconcito tranquile doode trbojg, $\mathbf{n}$ me intule aqii Usted tabe que soy micpe y

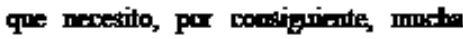

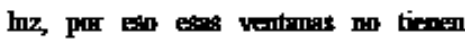
cartings y todv el que pasta cornsivern

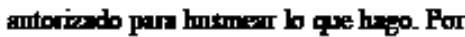
fortma yo wo veo. Pero hry algo per. Al

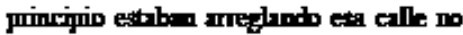

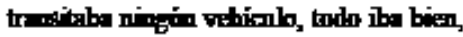

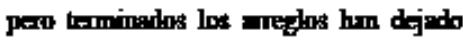
en el pavimanto, firate a frote de hos balcomes, ung arrista sobresoliente y tora no hy minje que yole: mftemaiticamente todbe vienten a chncri meris-mente thí, y a cadn instrate, mb mejor de unh frase, me hece taltar ed etruendo de $\mathrm{m}$ tirlemoto. $Y$ por este otro bato, en el affilto mojodo y resbalosin de h Alameda, Pilexe que vinieran a cre todos los colballos de

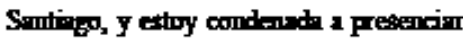
la botalidad de los cocheras, y w puedo 
चntorime y shlpo y les introlto y ellos me insultem a mi y talen mis sirvi-ntes a

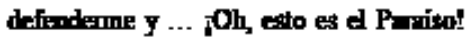
¡Y hahleme woted de los encrmios de la vida sminguim y hobleme de que yo debo quxier mocher a mi pás y consideri rmo bemanos a todos fios selvies infirives a las pobres bestirs que crtipm ihmm nontel

En los ojos que entmmabe la micpón

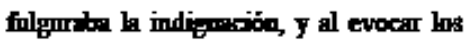
dnlores moidns de los pobnes minales la Intist sentín erizrise sn erquitita sensitilidad, esen sensitilind deticadn que

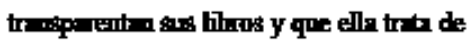

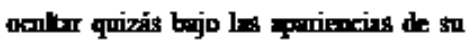
irtún.

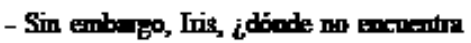
usted salvojes? iNo los ha hallodo en Fanis al ipgal que aqui?

-Pero allí und los poede hil ...

- Volrmos a su hib. Me intarese sobemm nofr Difime - si estro no es indieneción - ¿va a desmolle usted m teml pilleridy al de "Entre Dem Moodes'?

- No. "Fitre Dem Mondes" fine xálo un entryo. Ya counta a mis migps que b exribi vol mente parl patri mi exm?n de Evibitire Querig medir mis fiverses; oir lo que ha mís altp qútica emoper dijen de

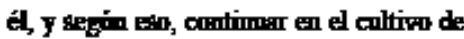
mi crescín $\mathrm{D}$ abodorrila de unn vez pam siempre.

- De mís estri jeguntar el resultado...

- Ya kn creb, prestr que roted me ve excribiands un mogr hibl. Lo que llevo publicado sim ens-yos, tínidns entryos,

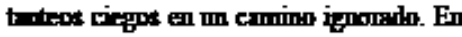

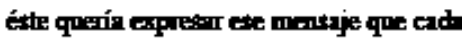
artista trwe al modo y por la expresión del cwl te hroh, se sufirs os more a veres.

- ¿Trabaja netrod metíxiçm*nlle?

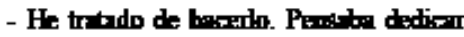
todss las mo inns extosivamente al

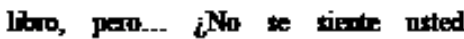

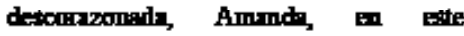
mbmontb? No me niegpe. A pes:ar de todo s. uptimisimo juveril, a despecho de todo lo que usted afimi de sn amr por los

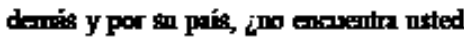
que aquí las indrivimalinhdes som

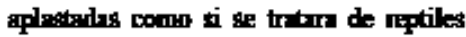

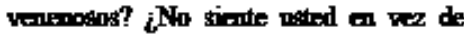
brazos que helory, manos crispades que trith de bajinly, de hecerta recondar en todo momento que antes que el ilenl 
deben este las comvariencips, hs prejuirios, el qué dir:in? Na me lo mifeves, prique no te lo cresil

- Ni tritr de negrilo, frés, pero neo ques, en Pinte, somes nesotrus mismos los allpobles de tal estado de cosen.

$-i \operatorname{Cimol}$

- porque tudns los atikts am los Wlest Cima, rmo los Dubli Unutig, las Silure Vildótaln, b Reber Matte, y Hiris, y Bacthens que repripitm el trimfo del Irte chlemoen ver de formar aquí un Imbiante, se vin a usufinctura del que ntros hxchanen por crear en la vigin Eircpal Y este cjomplo de hos pandes, es

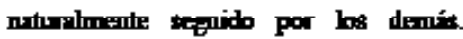
Pregumte woted a cada uno de los pi itites, de los poehs, de has mósicos mestros

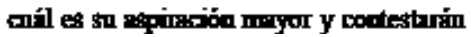
que es irte a Prís, no por m ento vigje, sinn par vivi y trabojir allí $\mathrm{Si}$ todos aquellos atistas trimfantes vivism entre nnsotrus, si trabjiken aqui, si monchierm squí sant grandes obros, veria notted cómo se forma el mbiente y se dejurl el goto en ptodo no distante

- Y mientras trmin, Amr ady, ¿qué bemos de hece nowotros?
- Prepiri el trilero pim los que hm de veri-

- ¡Oh, no! Yo no trenge instioto de apótbl

- Ya bimpoco; paril quibas que no,

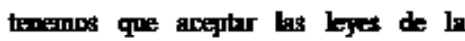
realinad

- In vida ded esfinitn es implacrble.

En este intrate entrí mon de bes bijes de

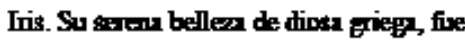
como ung clmidad dectur de la sela y sa sod rien como un ce icin sedante.

- Rebeca, dije yr, ti dentro de vinite añins neted comethat que hry en Smting un ambirente atistion y unn intensa vida expinitnal y usted encurntra en ellns m goce mudo mís refinndo y mít alto que todos lns que boy se le puede offect aquí, remende usted que sn montre ba

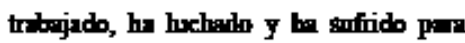
que usted porda sentir hs dnkanst de un vida que nosotros no comocinos.

A. LABARCA H

(Transcripciḱn: Anthen Matin Compos) 